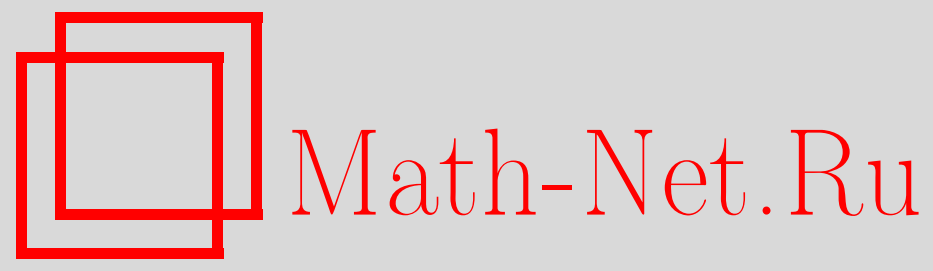

Ю. Э. Сеницкий, А. С. Ишутин, Общая устойчивость сжатых составных стержней переменного сечения, Вестн. Сам. гос. техн. ун-та. Сер. Физ.мат. науки, 2015, номер 2, 341-357

DOI: https://doi.org/10.14498/vsgtu1350

Использование Общероссийского математического портала MathNet.Ru подразумевает, что вы прочитали и согласны с пользовательским соглашением

http://www.mathnet.ru/rus/agreement

Параметры загрузки:

IP : 3.89 .197 .203

26 апреля 2023 г., 18:15:02

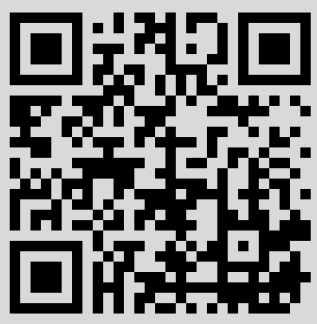


УДК 517.958:539.3(5)

\title{
ОБЩАЯ УСТОЙЧИВОСТЬ СЖАТЫХ СОСТАВНЫХ СТЕРЖНЕЙ ПЕРЕМЕННОГО СЕЧЕНИЯ
}

\author{
Ю. Э. Сеницкий, А. С. Ииутин
}

Самарский государственный архитектурно-строительный университет, Россия, 443001, Самара, ул. Молодогвардейская, 194.

\begin{abstract}
Аннотация
На основе технической теории стержней, кинематика деформирования которых дополнена деформациями поперечного сдвига, приводится точное решение задачи о потере устойчивости центрально сжатого составного стержня переменного сечения. Рассмотрен практически важный случай, соответствующий степенному закону изменения жесткости. В отличие от известных исследований, в работе используется прием, основанный на повышении порядка разрешающего дифференциального уравнения, что позволило в итоге получить новые результаты как для симметричных, так и для несимметричных форм потери устойчивости стержней при различных условиях их закрепления. Решения получены в цилиндрических, а для квадратичного закона изменения жесткости в элементарных функциях. Показано, что учет деформаций поперечного сдвига решетки при оценке общей устойчивости составных стержней переменного сечения является обязательным.
\end{abstract}

Ключевые слова: общая устойчивость, бифуркация, сквозной стержень, деформации сдвига, различные закрепления, неавтономные дифференциальные уравнения, повышение порядка, точное решение, коэффициенты приведенной длины.

doi: http://dx.doi.org/10.14498/vsgtu1350

Введение. Расчет на общую устойчивость стержней сплошного или составного переменного сечения на основе технической или уточненной (балка Тимошенко) теорий представляет интерес и в настоящее время, если иметь в виду построение замкнутых (или математическое обоснование) приближенных методов ее решения [1-9]. Следует отметить практическую значимость континуальной расчетной модели центрально-сжатого составного стержня переменного сечения, применяемой при расчете на общую устойчивость гибких дискретных стержневых систем башенного типа, в частности радио и телевизионных мачт, стрел кранов, буровых вышек, опор ЛЭП и эстакад $[5,10]$.

(C) 2015 Самарский государственный технический университет.

\section{Образец для цитирования}

С ен и цк и й Ю. Э., Ишу тин А. С. Общая устойчивость сжатых составных стержней переменного сечения // Вестн. Сам. гос. техн. ун-та. Сер. Физ.-мат. науки, 2015. Т. 19, № 2. C. 341-357. doi: 10.14498/vsgtu1350.

\section{Сведения об авторах}

Юрий Эдуардович Сеницкий (д.т.н., проф.; senitskiy@mail.ru; автор, ведущий переписку), заведующий кафедрой, каф. сопротивления материалов и строительной механики.

Александр Сергеевич Ииутин, студент, каф. сопротивления материалов и строительной механики. 
Вопросы обеспечения общей устойчивости таких систем при их проектировании играют важную роль в связи с применением в качестве материала для них высокопрочных сталей, а также легких сплавов, обладающих высокими деформативными свойствами. Условно сдвигово-изгибная расчетная модель стержня может успешно использоваться и при исследовании устойчивости плоских рам [11].

Наиболее полно устойчивость сплошных стержней переменного сечения со степенным законом изменения жесткости исследовалась А. Н. Динником [1], а в статье [4] приведены численные результаты расчетов для таких стержней. Подобная задача рассматривалась также в [2,3] и некоторых других. Вместе с тем следует отметить, что результаты этих исследований проведены без учета деформаций поперечного сдвига, играющих заметную роль именно в стержнях составного сечения. Исключением являются статьи одного из соавторов $[5,10,12]$, в которых получены замкнутые решения задач устойчивости для составных стержней с квадратичным законом изменения моментов инерции при их загружении полярными силами, а также приближенные решения для балки Тимошенко сплошного сечения [6-7].

В отличие от приведенных выше исследований, настоящая работа посвящена построению замкнутого (в пределах принятых допущений) решения задачи общей устойчивости для составного стержня переменного сечения. Для этой цели предлагается гипотетическая модель стержня сплошного сечения, эквивалентная по деформациям изгиба и единичным углам сдвига исследуемой сквозной стержневой конструкции. Существенным представляется и то, что в дальнейшем используется уточненная авторами модель С. П. Тимошенко. Такой подход позволил сформулировать критерий, при котором оказывается справедливой и традиционная схема учета деформаций сдвига.

Следует также подчеркнуть предлагаемую авторами настоящей работы корректную постановку рассматриваемой краевой задачи, основанную на дифференциальных уравнениях как 2-го, так и 4-го порядков, что при соответствующих граничных условиях позволило исследовать различные способы закрепления составного стержня, и не только симметричные, но и несимметричные формы потери устойчивости. В работе для этой цели используется прием повышения порядка разрешающего дифференциального уравнения. Вместе с тем, как отмечалось выше, авторы обычно пользовались лишь разрешающим уравнением 2-го порядка, что не позволило им исследовать все возможные формы потери устойчивости равновесия.

1. Постановка задачи. Рассматривается устойчивость центрально сжатого стержня переменного сечения (рис. 1). Сжимающая сила $P$ считается приложенной строго вдоль центральной оси стержня, которая остается прямолинейной вплоть до момента потери устойчивости (критического состояния $P_{\mathrm{cr}}$ ). Таким образом, потеря устойчивости сопровождается разветвлением форм равновесия (бифуркацией). Поскольку рассматриваемая ниже механическая система является консервативной, используется статический критерий потери устойчивости, основанный на уравнениях равновесия, составленных в деформированном состоянии стержня. При этом многопанельный составной стержень заменяется эквивалентным по единичным углам сдвига и изгибной жесткости стержнем сплошного сечения. Деформации поперечного сдвига в соответствии с гипотезой Энгессера-Тимошенко определяются законом из- 


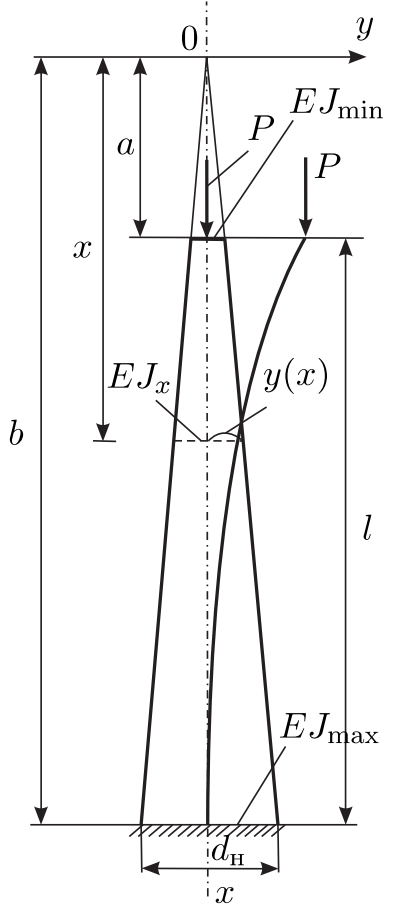

a

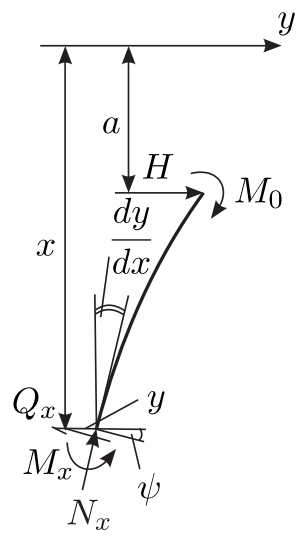

$b$

Рис. 1. Общая схема к задаче исследования устойчивости стержня переменного сечения

[Figure 1. The general scheme to the stability task of solving a rod of variable section]

менения перерезывающих сил, а деформации сжатия, ввиду их малости, не учитываются.

Как правило, ввиду постоянства поперечного сечения ветвей составных стержней с непрерывно изменяющейся по степенному закону изгибной жесткостью наибольший практический интерес представляет случай, когда

$$
A_{x}=A=\text { const }, \quad J_{x}(x)=J_{\max }(x / b)^{\alpha},
$$

где $A_{x}, J_{x}(x)$ - соответственно площадь и момент инерции поперечного сечения составного стержня. Остальные обозначения приведены на рис. 1.

Принимая во внимание сформулированные допущения, рассматриваемая задача представляет задачу о бифуркации изгибных форм равновесия.

Исходными соотношениями при этом являются уравнение совместности деформаций:

$$
\frac{d y}{d x}=\psi+\gamma
$$

физические уравнения, связывающие усилия и деформации:

$$
\begin{gathered}
E J_{x} \frac{d \psi}{d x}=-M_{x}, \\
\gamma=\frac{\mu}{G A} Q_{x}=\bar{\gamma} Q_{x}, \quad \bar{\gamma}=\frac{\mu}{G A}
\end{gathered}
$$


а также уравнения равновесия в деформированном состоянии стержня (рис. 1):

$$
\begin{gathered}
M_{x}=P y+H(x-a)+M_{0}, \\
Q_{x}=P \psi+H .
\end{gathered}
$$

Здесь $y, \psi, \gamma$-соответственно прогибы, углы поворота и углы сдвига в том же сечении стержня; $M_{x}, Q_{x}$ - изгибающий момент и перерезывающая сила; $H, M_{0}$ - реактивные усилия в сечении $x=a ; E, G$ - модули упругости и сдвига; $\mu, \bar{\gamma}$ - соответственно коэффициент неравномерности распределения касательных напряжений по поперечному сечению и единичный угол сдвига от $Q=1$.

В силу малости деформаций при составлении уравнений равновесия было принято (рис. $1, b): \sin \psi \cong \psi, \cos \psi \cong 1$. Дифференцируя равенство (2), находим

$$
E J_{x} \frac{d^{2} y}{d x^{2}}=E J_{x}\left(\frac{d \psi}{d x}+\frac{d \gamma}{d x}\right) .
$$

В результате подстановки в (7) продифференцированного соотношения (4), имея при этом в виду (6), получаем

$$
E J_{x} \frac{d^{2} y}{d x^{2}}=E J_{x}(1+\bar{\gamma} P) \frac{d \psi}{d x}
$$

Выражение (3) с учетом (5) принимает следующий вид:

$$
E J_{x} \frac{d \psi}{d x}=-\left(P y+H(x-a)+M_{0}\right) .
$$

Последнее равенство с учетом зависимости (1) представляет собой дифференциальное уравнение устойчивости составного стержня переменного сечения со степенным законом изменения жесткости при наличии деформаций сдвига (случай I):

$$
E J_{\max }(1+\bar{\gamma} P)^{-1}\left(\frac{x}{b}\right)^{\alpha} \frac{d^{2} y(x)}{d x^{2}}+P y(x)=-H(x-a)-M_{0} .
$$

Следует отметить, что приведенный выше вывод дифференциального уравнения устойчивости (10) основан на независимых (5) и (6) уравнениях равновесия.

Вместе с тем в обычной трактовке для этой цели вместо (6) используется известная зависимость Д. И. Журавского. С учетом (5) имеем

$$
Q_{x}=\frac{d M_{x}}{d x}=P \frac{d y}{d x}+H
$$

Повторяя рассуждения (7)-(9), при наличии (11) получаем в этом случае такое дифференциальное уравнение устойчивости (случай 프):

$$
E J_{\max }(1-\bar{\gamma} P)\left(\frac{x}{b}\right)^{\alpha} \frac{d^{2} y(x)}{d x^{2}}+P y=-H(x-a)-M_{0} .
$$


Добавляя к (10) или (12) граничные условия, определяемые соответствующими условиями закрепления концов стержня, получаем математическую формулировку рассматриваемой краевой задачи.

Легко установить связь между уравнениями (10) и (12). Действительно, из степенного разложения

$$
(1+\bar{\gamma} P)^{-1}=1-\bar{\gamma} P+(\bar{\gamma} P)^{2}-(\bar{\gamma} P)^{3}+\ldots
$$

замечаем, что $(1+\bar{\gamma} P)^{-1} \cong 1-\bar{\gamma} P$ при $\bar{\gamma} P \ll 1$. Таким образом, когда $\bar{\gamma} P \ll 1$, уравнение (10) тождественно совпадает с (12).

Следует обратить внимание, что уравнениям (10) и (12) соответствует одно однородное дифференциальное уравнение

$$
b^{2-\alpha} x^{\alpha} \frac{d^{2} y}{d x^{2}}+\omega y=0,
$$

в котором используются различные критические параметры $\omega$ :

$$
\begin{gathered}
\omega=P b^{2}(1+\bar{\gamma} P)\left(E J_{\max }\right)^{-1} \quad(\text { в случае I }), \\
\left.\omega=P b^{2}\left((1-\bar{\gamma} P)^{-1}\left(E J_{\max }\right)\right)^{-1} \quad \text { (в случае } \amalg\right) .
\end{gathered}
$$

2. Метод решения. Решение задачи осуществляется непосредственным интегрированием уравнения (10) или (12). Известно, что общее решение линейного неоднородного дифференциального уравнения с переменными коэффициентами может быть представлено в виде суммы:

$$
y(x)=y_{1}(x)+y_{2}(x),
$$

где $y_{1}(x)$ - общее решение соответствующего (10) или (12) однородного уравнения, а $y_{2}(x)$ - какое-либо частное решение неоднородного уравнения (10).

Общий интеграл однородного уравнения

$$
\frac{d^{2} y_{1}(x)}{d x^{2}}+b^{\alpha-2} \omega x^{-\alpha} y_{1}(x)=0
$$

можно получить в виде

$$
y_{1}(x)=C_{1} \sqrt{x} J_{\nu}(\bar{x})+C_{2} \sqrt{x} Y_{\nu}(\bar{x}),
$$

где

$$
\bar{x}=\frac{1}{k} \omega^{1 / 2}\left(\frac{x}{b}\right)^{k}, \quad k=\frac{2-\alpha}{2}, \quad \nu=|2-\alpha|^{-1}
$$

$J_{\nu}(\bar{x}), Y_{\nu}(\bar{x})$ - цилиндрические функции (функции Бесселя) 1-го и 2-го рода порядка $\nu$, а $C_{1}, C_{2}$ - постоянные интегрирования.

Частное решение $y_{2}(x)$ определяется по виду правой части $(10)$ :

$$
y_{2}(x)=-\frac{1}{P}\left(H(x-a)+M_{0}\right) .
$$


Складывая (17) и (19) в соответствии с (15), окончательно имеем

$$
y(x)=C_{1} \sqrt{x} J_{\nu}(\bar{x})+C_{2} \sqrt{x} Y_{\nu}(\bar{x})-\frac{1}{P}\left(H(x-a)+M_{0}\right),
$$

при этом $H$ и $M_{0}$ определяются условиями закрепления верхнего конца стержня.

Следует отметить особый случай $\alpha=2$, соответствующий квадратичному закону изменения жесткости (1), при котором согласно (18) $\nu \rightarrow \infty$ и, естественно, полученное выше общее решение (17) и (20) не проходит. Рассмотрим этот случай отдельно.

При $\alpha=2$ уравнение (16) представляет собой уравнение Эйлера

$$
\frac{d^{2} y_{1}(x)}{d x^{2}}+\omega x^{-2} y_{1}(x)=0,
$$

решение которого записывается в виде

$$
\bar{y}_{1}(x)=\sqrt{x}\left(C_{1} \cos \bar{x}_{1}+C_{2} \sin \bar{x}_{1}\right),
$$

где

$$
\bar{x}_{1}=\frac{\sqrt{4 \omega-1}}{2} \ln x .
$$

При этом критический параметр $\omega>0.25$, поскольку случай $\omega<0.25$ соответствуют нереально большим гибкостям стержня.

Частное решение неоднородного уравнения (10) в случае $\alpha=2$ определяется по формуле (19), а его общее решение в соответствии с (15), (19), (21) равенством

$$
y(\bar{x})=\sqrt{x}\left(C_{1} \cos \bar{x}_{1}+C_{2} \sin \bar{x}_{1}\right)-\frac{1}{P}\left(H(x-a)+M_{0}\right) .
$$

В дальнейшем искомым является критический параметр $\omega_{\mathrm{cr}}$, зная который, сжимающая критическая сила может быть определена из соотношений (13) или (14) (случаи I и II).

Случай I. Из равенства (13) следует квадратное уравнение [13], корни которого

$$
P_{\mathrm{cr}}=-\frac{1}{2 \bar{\gamma}} \pm \sqrt{\frac{1}{4 \bar{\gamma}^{2}}+\frac{E J_{\max }}{\bar{\gamma} b^{2}} \omega_{\mathrm{cr}}},
$$

или в более удобной форме:

$$
P_{\mathrm{cr}}=\frac{\pi^{2} E J_{\max }}{\left(\beta_{1} l\right)^{2}}
$$

где $l$ - длина стержня (см. рис. 1$) ; \beta_{1}$ - коэффициент приведенной длины стержня:

$$
\beta_{1}=\beta \sqrt{\omega_{\mathrm{cr}} E J_{\max } b^{-2}\left[\left(\frac{1}{4 \bar{\gamma}^{2}}+\frac{\omega_{\mathrm{cr}} E J_{\max }}{\bar{\gamma} b^{2}}\right)^{1 / 2}-\frac{1}{2 \bar{\gamma}}\right]^{-1}} .
$$


Здесь

$$
\beta=\frac{\pi}{1-k} \frac{1}{\sqrt{\omega_{\mathrm{cr}}}}, \quad k=\frac{a}{b},
$$

причем $\beta$ - безразмерный коэффициент приведенной длины, определяемый без учета деформаций сдвига решетки [4]. Вид $\omega_{\mathrm{cr}}=\omega_{\mathrm{cr}}(l)$ будет определен ниже.

Случай ㅍ. Аналогично из (14) имеем

$$
P_{\mathrm{cr}}=\frac{\omega_{\mathrm{cr}} E J_{\max }}{b^{2}+\bar{\gamma} E J_{\max } \omega_{\mathrm{cr}}} \quad \text { или } \quad P_{\mathrm{cr}}=\frac{\pi^{2} E J_{\max }}{\left(\beta_{1} l\right)^{2}} \text {, }
$$

где

$$
\beta_{1}=\frac{\pi}{l}\left[b^{2}+\omega_{\mathrm{cr}} \bar{\gamma} E J_{\max }\right]^{-1 / 2} .
$$

Здесь также $\omega_{\mathrm{cr}}=\omega_{\mathrm{cr}}(l)$.

3. Решение в случае симметричной формы потери устойчивости. Поскольку кривая, определяющая форму потери устойчивости в зависимости от условий закрепления стержня, должна удовлетворять четырем граничным условиям (по два на каждом конце стержня), полученное выше решение (20) или (23) в общем случае при наличии двух произвольных постоянных $C_{1}$ и $C_{2}$ не может это обеспечить, поэтому оно должно быть расширено.

Следует отметить, что равенства (20) или (23) можно использовать при исследовании симметричных форм потери устойчивости стержней переменного сечения. Естественно, сам стержень при этом должен быть конструктивно симметричным (рис. 2).

В дальнейшем понадобится выражение для углов наклона касательной к деформированной оси стержня, определяющей форму потери устойчивости. Дифференцируя выражения (17) и (21) в случае $\alpha \neq 2$, получим

$$
\begin{aligned}
\frac{d y_{1}(x)}{d x}=C_{1}\left\{\frac{1}{2} x^{-1 / 2}\right. & \left.J_{n}(\bar{x})+x^{1 / 2}\left[\frac{n}{\bar{x}} J_{n}(\bar{x})-J_{n+1}(\bar{x})\right] \frac{d \bar{x}}{d x}\right\}+ \\
+ & C_{2}\left\{\frac{1}{2} x^{-1 / 2} Y_{n}(\bar{x})+x^{1 / 2}\left[\frac{n}{\bar{x}} Y_{n}(\bar{x})-Y_{n+1}(\bar{x})\right] \frac{d \bar{x}}{d x}\right\},
\end{aligned}
$$

где

$$
\frac{d \bar{x}}{d x}=\omega^{1 / 2}\left(\frac{x}{a}\right)^{(1-2 n) / 2 n} \frac{1}{a} .
$$

А в случае $\alpha=2$ получим

$$
\begin{aligned}
\frac{d y_{1}(x)}{d x}=C_{1}\left[\frac{1}{2} x^{-1 / 2} \cos \bar{x}_{1}-x^{1 / 2} \sin \bar{x}_{1} \frac{d \bar{x}_{1}}{d x}\right]+ & \\
& +C_{2}\left[x^{-1 / 2} \sin \bar{x}_{1}+x^{1 / 2} \cos \bar{x}_{1} \frac{d \bar{x}_{1}}{d x}\right]
\end{aligned}
$$

причем

$$
\frac{d \bar{x}_{1}}{d x}=\frac{1}{2}(4 \omega-1)^{1 / 2} \frac{1}{x}
$$




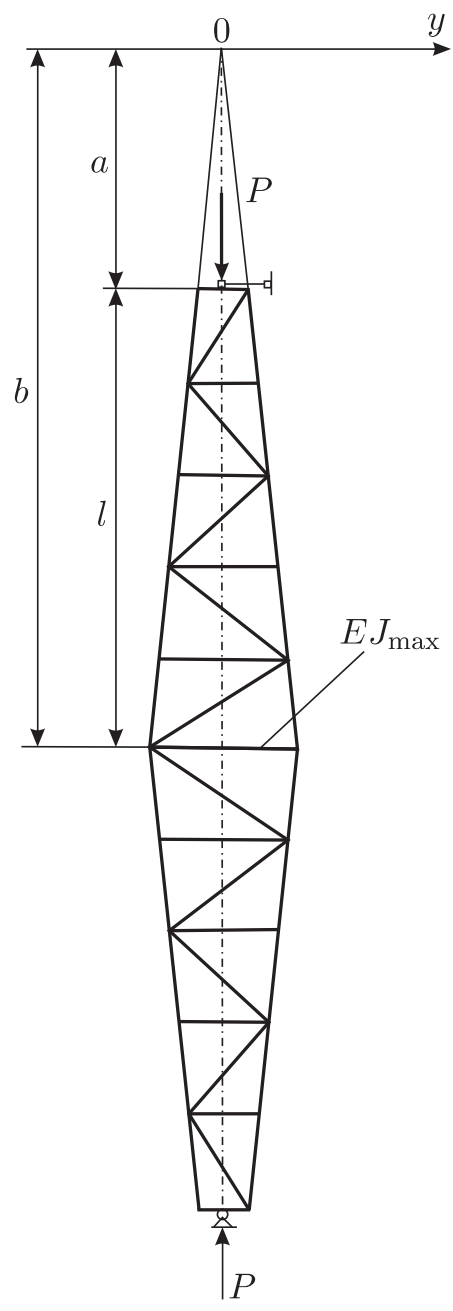

Рис. 2. Расчетная схема стержня при симметричных формах потери устойчивости [Figure 2. The design scheme for a rod with symmetric cases buckling]

Рассмотрим ниже некоторые примеры расчета.

1. Симметричный составной стержень при произвольном $\alpha \neq 2$ шарнирно закреплен по концам. Соответствующими граничными условиями при этом являются условия симметрии формы потери устойчивости (рис. 2):

$$
y_{1}(a)=0 ;\left.\quad \frac{d y_{1}}{d x}\right|_{x=b}=0 .
$$

Подстановка равенств (17), (28) в соотношения (31) формирует следующую однородную систему алгебраических уравнений для $C_{1}$ и $C_{2}$ :

$$
\begin{aligned}
& C_{1} J_{n}(\bar{a})+C_{2} Y_{n}(\bar{a})=0, \\
& C_{1}\left\{\left[\frac{1}{2} b^{-1 / 2}+b^{1 / 2} \frac{n}{\bar{b}}\right] J_{n}(\bar{b})-b^{1 / 2} J_{n+1}(\bar{b})\right\}+ \\
& \quad+C_{2}\left\{\left[\frac{1}{2} b^{-1 / 2}+b^{1 / 2} \frac{n}{\bar{b}}\right] Y_{n}(\bar{b})-b^{1 / 2} Y_{n+1}(\bar{b})\right\}=0 .
\end{aligned}
$$


Здесь

$$
\bar{a}=\frac{2}{2-\alpha} \omega^{1 / 2}, \quad \bar{b}=\frac{2}{2-\alpha} \omega^{1 / 2}(b / a)^{(2-\alpha) / 2}, \quad n=\frac{1}{|2-\alpha|} .
$$

Разыскивая нетривиальные решения для $C_{1}$ и $C_{2}$, приравниваем главный детерминант системы (32) к нулю. В результате получаем

$$
\begin{aligned}
J_{n}(\bar{a})\left[\left(\frac{1}{2} b^{-1 / 2}+b^{1 / 2} \frac{n}{\bar{b}}\right)\right. & \left.Y_{n}(\bar{b})-b^{1 / 2} Y_{n+1}(\bar{b})\right]= \\
& =Y_{n}(\bar{a})\left[\left(\frac{1}{2} b^{-1 / 2}+b^{1 / 2} \frac{n}{\bar{b}}\right) J_{n}(\bar{b})-b^{1 / 2} J_{n+1}(\bar{b})\right] .
\end{aligned}
$$

Трансцендентное уравнение устойчивости (34) позволяет определить итерационным путем наименьший корень $\omega_{\min }$ - критический параметр, а затем по формулам (26), (24) - коэффициент приведенной длины $\beta_{1}$ и критическую силу $P_{\text {cr. }}$.

2. Аналогичное решение задачи в случае $\alpha=2$ при наличии равенств $(22)$, (29) и (31) приводит к соотношениям

$$
C_{1} \cos \bar{a}_{1}+C_{2} \sin \bar{a}_{1}=0 \quad \text { при } x=a,
$$

$C_{1}\left[\cos \bar{b}_{1}-\frac{1}{2}(4 \omega-1)^{1 / 2} \sin \bar{b}_{1}\right]+C_{2}\left[\sin \bar{b}_{1}+\frac{1}{2}(4 \omega-1)^{1 / 2} \cos \bar{b}_{1}\right] \quad$ при $x=b$,

где $\bar{a}_{1}, \bar{b}_{1}$ определяются по формулам (33).

Трансцендентное уравнение для определения $\omega_{\min }$ принимает вид

$$
\cos \bar{a}_{1}\left[\sin \bar{b}_{1}+\frac{1}{2}(4 \omega-1)^{1 / 2} \cos \bar{b}_{1}\right]=\sin \bar{a}_{1}\left[\cos \bar{b}_{1}-\frac{1}{2}(4 \omega-1)^{1 / 2} \sin \bar{b}_{1}\right]
$$

и уточняет соответствующее ошибочное уравнение, приведенное в монографии [3].

3. Рассмотрим составной стержень, жестко защемленный по концам при $\alpha \neq 2$. Из-за симметрии формы потери устойчивости граничные условия запишутся следующим образом:

$$
\left.\frac{d y_{1}}{d x}\right|_{x=a}=\left.\frac{d y_{1}}{d x}\right|_{x=b}=0 .
$$

В результате подстановки выражения (28) в (35) получаем однородную систему уравнений:

$$
\begin{aligned}
C_{1}\left[\left(\frac{1}{2} a^{-1 / 2}+\right.\right. & \left.\left.a^{1 / 2} \frac{n}{\bar{a}}\right) J_{n}(\bar{a})-a^{1 / 2} J_{n+1}(\bar{a})\right]+ \\
& +C_{2}\left[\left(\frac{1}{2} a^{-1 / 2}+a^{1 / 2} \frac{n}{\bar{a}}\right) Y_{n}(\bar{a})-a^{1 / 2} Y_{n+1}(\bar{a})\right]=0, \\
C_{1}\left[\left(\frac{1}{2} b^{-1 / 2}+\right.\right. & \left.\left.b^{1 / 2} \frac{n}{\bar{a}}\right) J_{n}(\bar{b})-b^{1 / 2} J_{n+1}(\bar{b})\right]+ \\
& +C_{2}\left[\left(\frac{1}{2} b^{-1 / 2}+b^{1 / 2} \frac{n}{\bar{b}}\right) Y_{n}(\bar{b})-b^{1 / 2} Y_{n+1}(\bar{b})\right]=0,
\end{aligned}
$$


откуда затем следует трансцендентное уравнение для определения $\omega_{\min }$ :

$$
\begin{aligned}
& {\left[\left(\frac{1}{2} a^{-1 / 2}+a^{1 / 2} \frac{n}{\bar{a}}\right) J_{n}(\bar{a})-a^{1 / 2} J_{n+1}(\bar{a})\right]\left[\left(\frac{1}{2} b^{-1 / 2}+b^{1 / 2} \frac{n}{\bar{b}}\right) Y_{n}(\bar{b})-b^{1 / 2} Y_{n+1}(\bar{b})\right]=} \\
= & {\left[\left(\frac{1}{2} b^{-1 / 2}+b^{1 / 2} \frac{n}{\bar{b}}\right) J_{n}(\bar{b})-b^{1 / 2} J_{n+1}(\bar{b})\right]\left[\left(\frac{1}{2} a^{-1 / 2}+a^{1 / 2} \frac{n}{\bar{a}}\right) Y_{n}(\bar{a})-a^{1 / 2} Y_{n+1}(\bar{a})\right] . }
\end{aligned}
$$

Здесь $\bar{a}, \bar{b}$ и $n$ определяются по формулам (33).

4. В случае $\alpha=2$ по той же схеме с использованием равенств (30), (35) (соответственно при $x=a$ и $x=b$ ) получаем

$$
\begin{aligned}
& C_{1}\left[\cos \bar{a}_{1}-\frac{1}{2}(4 \omega-1)^{1 / 2} \sin \bar{a}_{1}\right]+C_{2}\left[\sin \bar{a}_{1}+\frac{1}{2}(4 \omega-1)^{1 / 2} \cos \bar{a}_{1}\right]=0, \\
& C_{1}\left[\cos \bar{b}_{1}-\frac{1}{2}(4 \omega-1)^{1 / 2} \sin \bar{b}_{1}\right]+C_{2}\left[\sin \bar{b}_{1}+\frac{1}{2}(4 \omega-1)^{1 / 2} \cos \bar{b}_{1}\right]=0 .
\end{aligned}
$$

Приравнивая главный детерминант этой системы к нулю, получаем уравнение для определения $\omega_{\min }$ :

$$
\begin{aligned}
& {\left[\cos \bar{a}_{1}-\frac{1}{2}(4 \omega-1)^{1 / 2} \sin \bar{a}_{1}\right]\left[\sin \bar{b}_{1}+\frac{1}{2}(4 \omega-1)^{1 / 2} \cos \bar{b}_{1}\right]=} \\
& \quad=\left[\cos \bar{b}_{1}-\frac{1}{2}(4 \omega-1)^{1 / 2} \sin \bar{b}_{1}\right]\left[\sin \bar{a}_{1}+\frac{1}{2}(4 \omega-1)^{1 / 2} \cos \bar{a}_{1}\right] .
\end{aligned}
$$

Равенство (36) уточняет соответствующее ошибочное уравнение, приведенное в монографии [3].

4. Обобщение решения рассматриваемой краевой задачи. На основе решений (17), (21) разрешающего дифференциального уравнения втрого порядка (10), (12), были сформулированы (п. 3) трансцендентные уравнения устойчивости для критического параметра $\omega_{\mathrm{cr}}=\omega_{\min }$ центрально сжатого составного стержня со степенным законом изменения жёсткости. При этом получены результаты для заранее (априори) известных симметричных форм потери устойчивости.

Однако в случае произвольных несимметричных форм исследовать устойчивость составного стержня, пользуясь соотношениями (17), (21), не представляется возможным из-за переопределённости системы граничных условий (по два условия на каждом конце для всех схем закрепления стержня). Для этой цели предложена методика, связанная с повышением порядка разрешающего дифференциального уравнения и дальнейшей корректной постановкой рассматриваемой краевой задачи.

Дифференцируя дважды уравнения (10) и (12), получаем

$$
y^{(4)}(x)+\frac{2 \alpha}{x} y^{\prime \prime \prime}(x)+\left[\frac{\alpha(\alpha-1)}{x^{2}}+\frac{\omega}{x^{\alpha}}\right] y^{\prime \prime}(x)=0 .
$$

Здесь в соответствии с (13), (14) $\omega$ представляет собой критический параметр преобразованных уравнений (10) и (12):

$$
\omega=\frac{P b^{\alpha}}{E J_{\max }}\left\{\begin{array}{l}
(1+\bar{\gamma} P) \\
(1-\bar{\gamma} P)^{-1}
\end{array}\right\} \text {. }
$$


В дальнейшем будем рассматривать квадратичный закон изменения жёсткости стержня $(\alpha=2)$. Построим для него новое замкнутое решение задачи.

При $\alpha=2$ дифференциальное уравнение (37) принимает вид

$$
y^{(4)}(x)+\frac{4}{x} y^{\prime \prime \prime}(x)+\frac{2+\omega}{x^{2}} y^{\prime \prime}(x)=0 .
$$

Введём новую функцию

$$
y^{\prime \prime}(x)=z(x)
$$

и запишем уравнение (38) так:

$$
z^{\prime \prime}(x)+\frac{4}{x} z^{\prime}(x)+\frac{2+\omega}{x^{2}} z(x)=0 .
$$

Общее решение уравнения (40) имеет вид

$$
z(\bar{x})=\exp (-n \bar{x})\left[C_{1} \cos m \bar{x}+C_{2} \sin m \bar{x}\right]
$$

где $\bar{x}=\ln x$,

$$
n=\frac{3}{2}, \quad m=\frac{1}{2}(4 \omega-1)^{1 / 2},
$$

$\omega>1 / 4$. Случай $\omega<1 / 4$ соответствует нереально большим гибкостям стержня и в дальнейшем не рассматривается.

Из дифференциального уравнения (39) находим

$$
\begin{aligned}
& y^{\prime \prime \prime}(x)=\exp [-(1+n) \bar{x}]\left[-C_{1}(n \cos m \bar{x}+m \sin m \bar{x})+\right. \\
& \left.+C_{2}(\cos m \bar{x}-n \sin m \bar{x})\right] \\
& y^{\prime}(x)=\left[1+\frac{(1-n)^{2}}{m^{2}}\right]^{-1} m^{-1}\left[C_{1}\left(\sin m \bar{x}+\frac{1-n}{m} \cos m \bar{x}\right)+\right. \\
& \left.+C_{2}\left(\frac{1-n}{m} \sin m \bar{x}-\cos m \bar{x}\right)\right]+C_{3}, \\
& y(x)=\left[1+\frac{(1-n)^{2}}{m^{2}}\right]^{-1}\left[1-\frac{(2-n)^{2}}{m^{2}}\right]^{-1} m^{-1} \times \\
& \times\left\{C_{1}\left[(\sin m \bar{x}-\cos m \bar{x})+\frac{2-n}{m}(\sin m \bar{x}+\cos m \bar{x})\right]+\right. \\
& \left.+C_{2}\left[(\sin m \bar{x}-\cos m \bar{x})+\frac{2-n}{m}(\cos m \bar{x}+\sin m \bar{x})\right]+C_{3} x+C_{4}\right\} .
\end{aligned}
$$

Соотношения (41) и (43) представляют собой решение рассматриваемой задачи, которое справедливо для произвольных условий закрепления составного стержня с квадратичным законом изменения жёсткости.

Добавляя в каждом конкретном случае однородные граничные условия, получаем соответствующую краевую задачу на собственные значения.

Ниже сформулируем уравнения устойчивости для различных условий закрепления составного стержня при $\alpha=2$. 
1. Шарнирное закрепление обоих концов. В этом случае на обоих концах перемещения и моменты равны нулю. Принимая во внимание соотношения (3) и (8), имеем граничные условия

$$
y(a)=y(b)=0,\left.\quad y^{\prime \prime}\right|_{x=a}=\left.y^{\prime \prime}\right|_{x=b}=0 .
$$

Подставляя выражения (41), (43) в равенства (44), получаем однородную систему алгебраических уравнений относительно постоянных $C_{1}, C_{2}, C_{3}, C_{4}$. Разыскивая затем её нетривиальные решения, приравниваем нулю главный детерминант. В результате получаем

$$
\left|\begin{array}{cccc}
{[B(\omega, \bar{a})+D(\omega, \bar{a})]} & {[B(\omega, \bar{a})+D(\omega, \bar{a})]} & E(\omega) a & E(\omega) \\
\cos m \bar{a} & \sin m \bar{a} & 0 & 0 \\
{[B(\omega, \bar{b})+D(\omega, \bar{b})]} & {[B(\omega, \bar{b})+D(\omega, \bar{b})]} & E(\omega) b & E(\omega) \\
\cos m \bar{b} & \sin m \bar{b} & 0 & 0
\end{array}\right|=0
$$

Здесь

$$
\begin{gathered}
B(\omega, \bar{x})=\sin m \bar{x}-\cos m \bar{x}, \quad D(\omega, \bar{x})=\frac{2-n}{m}(\sin m \bar{x}+\cos m \bar{x}), \\
E(\omega)=\left[1+\frac{(1-n)^{2}}{m^{2}}\right]\left[1+\frac{(2-n)^{2}}{m^{2}}\right] m,
\end{gathered}
$$

где $n, m$ вычисляются по формулам (42), а $\bar{a}$ и $\bar{b}$ находятся из равенств

$$
\bar{a}=\ln a, \quad \bar{b}=\ln b .
$$

Трансцендентное уравнение устойчивости (45) решается итерационным путём. Разыскивается минимальный корень $\omega_{\min }=\omega_{\mathrm{cr}}$, а затем из равенств (25), (26) и (24) - коэффициенты приведенной длины и критическая нагрузка.

2. Жёсткое защемление обоих концов. В этом случае граничные условия формулируются следующем образом:

$$
y(a)=y(b)=0,\left.\quad y^{\prime}(x)\right|_{x=a}=\left.y^{\prime}(x)\right|_{x=b}=0 .
$$

Подставляя выражения (43) в равенства (47), получаем однородную систему, приравниваем нулю главный детерминант и получаем трансцендентное уравнение устойчивости для $\omega_{\min }=\omega_{\mathrm{cr}}$ :

$$
\left|\begin{array}{cccc}
{[B(\omega, \bar{a})+D(\omega, \bar{a})]} & {[B(\omega, \bar{a})+D(\omega, \bar{a})]} & E(\omega) a & E(\omega) \\
F(\omega, \bar{a}) & G(\omega, \bar{a}) & H(\omega) & 0 \\
{[B(\omega, \bar{b})+D(\omega, \bar{b})]} & {[B(\omega, \bar{b})+D(\omega, \bar{b})]} & E(\omega) b & E(\omega) \\
F(\omega, \bar{b}) & G(\omega, \bar{b}) & H(\omega) & 0
\end{array}\right|=0
$$

где

$$
\begin{gathered}
F(\omega, \bar{x})=\sin m \bar{x}+\frac{1-n}{m} \cos m \bar{x}, \quad G(\omega, \bar{x})=\frac{1-n}{m} \sin m \bar{x}-\cos m \bar{x} \\
H(\omega)=\left[1+\frac{(1-n)^{2}}{m^{2}}\right] m .
\end{gathered}
$$


3. Консольный стержень. Граничные условия задачи для этого случая имеют вид

$$
y^{\prime \prime}(a)=y^{\prime \prime \prime}(a)=0, \quad y(b)=y^{\prime}(b)=0 .
$$

Используя выражения (42), (43) и (49), в соответствии с описанной выше процедурой получаем следующее трансцендентное уравнение устойчивости для критического параметра $\omega_{\min }=\omega_{\mathrm{cr}}$ :

$$
\left|\begin{array}{cccc}
\cos m \bar{a} & \sin m \bar{a} & 0 & 0 \\
K(\omega, \bar{a}) & L(\omega, \bar{a}) & 0 & 0 \\
{[B(\omega, \bar{b})+D(\omega, \bar{b})]} & {[B(\omega, \bar{b})+D(\omega, \bar{b})]} & E(\omega) b & E(\omega) \\
F(\omega, \bar{b}) & G(\omega, \bar{b}) & H(\omega) & 0
\end{array}\right|=0 .
$$

Здесь $K(\omega, \bar{x})=n \cos m \bar{x}+m \sin m \bar{x}, L(\omega, \bar{x})=n \sin m \bar{x}-m \cos m \bar{x}$.

4. Случай, когда один конец защемлён, а другой шарнирно закреплён. Граничные условия формулируются следующим образом:

$$
\begin{aligned}
& y(a)=y^{\prime \prime}(a)=0, \\
& y(b)=y^{\prime}(b)=0 .
\end{aligned}
$$

Применяя описанный выше алгоритм, получаем следующее уравнение устойчивости для $\omega_{\min }=\omega_{\mathrm{cr}}$ :

$$
\left|\begin{array}{cccc}
{[B(\omega, \bar{a})+D(\omega, \bar{a})]} & {[B(\omega, \bar{a})+D(\omega, \bar{a})]} & E(\omega) a & E(\omega) \\
\cos m \bar{a} & \sin m \bar{a} & 0 & 0 \\
{[F(\omega, \bar{b})+D(\omega, \bar{b})]} & {[G(\omega, \bar{b})+D(\omega, \bar{b})]} & E(\omega) b & E(\omega) \\
F(\omega, \bar{b}) & G(\omega, \bar{b}) & H(\omega) & 0
\end{array}\right|=0 .
$$

Таким образом, в зависимости от условий закрепления стержня критический параметр $\omega_{\text {cr }}$ является наименьшим корнем одного из полученных трансцендентных уравнений (45), (48), (50), (51).

5. Численный анализ результатов. Исходными данными для проведения расчётов устойчивости центрально-сжатого составного стержня переменного сечения с квадратичным законом изменения жёсткости $\alpha=2$ являются модуль упругости материала $E$, площадь поперечного сечения ветви $A_{b}$, а также соотношения площадей поперечных сечений горизонтальных поясов и раскосов $A_{n} / A_{b}$ и $A_{p} / A_{b}$ решётчатого стержня. Известными считаются $J_{\max }, l$ (рис. 1) размеры $d_{H}, d_{b}$ и число панелей $i$ многопанельного стержня.

С использованием формулы (1) при $x=a$ и соотношения (46) вычисляются параметры $a, b, \bar{a}, \bar{b}$ :

$$
a=l \frac{d_{b}}{d_{H}-d_{b}}, \quad b=l+a, \quad \bar{a}=\ln a, \quad \bar{b}=\ln b .
$$

Единичные углы сдвига определяются для каждой панели стержня (рис. 3) из соотношения

$$
\bar{\gamma}_{i} \approx \operatorname{tg} \bar{\gamma}_{i}=\frac{\delta_{11}}{(l / i)}
$$

где $\delta_{11}$ - единичное перемещение по направлению $\bar{Q}$. За расчётное $\bar{\gamma}_{i}$ принимается среднее арифметическое значение. 


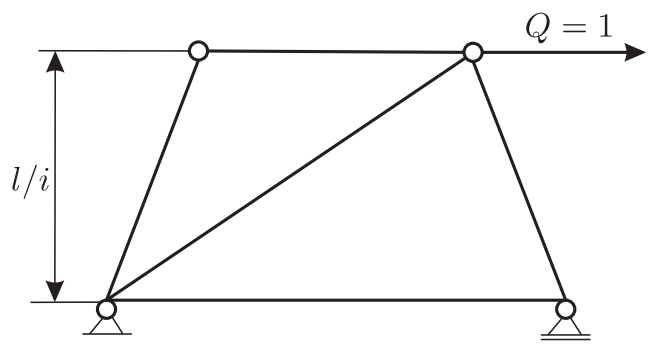

Рис. 3. Схема к определению единичных углов сдвига для панели

[Figure 3. The design scheme for the definition of angular shift for a panel]

В зависимости от способов закрепления обоих концов стержня итерационным методом решается соответствующее трансцендентное уравнение (45), (48), (50) или (51). Для этой цели задаёмся $\omega_{k}$ и, варьируя его, добиваемся удовлетворения указанных уравнений.

Определив наименьший корень $\omega_{\mathrm{kp}}=\omega_{\min }$ по формулам (25) или $(27)$, вычисляем коэффициент приведённой длины $\beta$ или $\beta_{1}$, а затем из выражения (24) определяем критическую силу $P_{\text {cr }}$.

В качестве модельного примера рассматривается десятипанельный стальной стержень решётчатого типа пролётом $l=2.0 \mathrm{M}, d=0.2 \mathrm{м}$ и $d_{b}=0.045 \mathrm{м}$. Каждая из четырёх ветвей стержня трубчатого сечения диаметром $0.01 \mathrm{~m}$ с толщиной стенки $\Delta=0.001$ м. Другие исходные данные: $E=2.1 \cdot 10^{11} \mathrm{H} / \mathrm{m}^{2}$, $A_{b}=0.126 \cdot 10^{-3} \mathrm{~m}^{2}, J_{\max }=0.126 \cdot 10^{-5} \mathrm{~m}^{4}, A_{p} / A_{b}=0.1, A_{n} / A_{b}=0.2$ $\left(A_{b}, A_{p}, A_{n}\right.$ - соответственно площади поперечных сечений ветвей, раскосов и поясов). Для указанного типа решётки единичный угол сдвига составил $\bar{\gamma}=(4.51 / E) \cdot 10^{3} \mathrm{H}^{-1}$.

На рис. 4 приведены графики коэффициента приведенной длины $\beta$ для составного стержня $(\alpha=2)$ при различных условиях закрепления его концевых сечений, полученные в результате варьирования деформаций сдвига решётки $\bar{\gamma}$. Кривые 1-4 соответствуют консольному, шарнирному, жёстко защемлённому и смешанному способам закрепления соответственно.

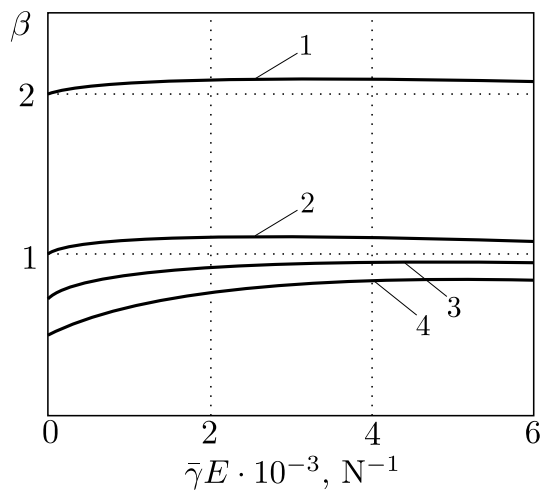

Рис. 4. Значения коэффициента приведенной длины $\beta$ для составного стержня $(\alpha=2)$ для различных условий закрепления: 1 - консольное; 2 - шарнирное; 3 -жёсткое; 4 -смешанное [Figure 4. The values of the coefficient $\beta$ for the composite rod $(\alpha=2)$ under different conditions of fixing: 1 - single-sided support; 2 - hinge support; 3 - rigid fixing; 4 - combined fixing] 
Следует отметить, что значения $\beta$ превышают аналогичные значения коэффициентов приведенной длины, подсчитанные при $\bar{\gamma}=0$. При этом влияние деформаций сдвига решётки на $\beta$ возрастает с увеличением жёсткости закрепления концов стержня и становится максимальным для жёстко защемлённого стержня. В отличие от стержней сплошного сечения, при оценке устойчивости составных стержней учёт деформаций сдвига решётки является необходимым.

В заключение можно сделать следующие выводы.

1. Сформулирована краевая задача устойчивости для центрально-сжатых составных стержней переменного сечения со степенным законом изменения жесткости, основанная на уточненных авторами соотношениях теории С. П. Тимошенко.

2. Получены впервые обобщенные трансцендентные уравнения устойчивости справедливые для исследования как симметричных, так и несимметричных форм равновесия составных стержней переменного сечения.

3. Показано, что для рассматриваемых стержней деформации поперечного сдвига решетки оказывают заметное влияние на окончательные результаты и должны учитываться при проведении расчётов в прикладных задачах.

\section{ORCID}

Юрий Эдуардович Сеницкий: http://orcid.org/0000-0002-0663-5563

\section{БИБЛИОГРАФИЧЕСКИЙ СПИСОК}

1. Динник А. Н. Продольный изгиб. Теория и приложения. М., Л.: ГОНТИ, 1939. 238 с.

2. Тимошенко С. П. Устойчивость упругих систем. М.: ГИТТЛ, 1955. 568 с.

3. Вольмир А. С. Устойчивость деформированных систем. М.: Наука, 1967. 984 с.

4. Лейтес С. Д. Устойчивость сжатых стержней, жёсткость которых изменяется по степенному закону / Материалы по металлическим конструкииям. М.: Стройиздат, 1962. C. $13-74$.

5. Сеницкий Ю. Э. Устойчивость сквозных стоек переменного сечения, загруженных полярными силами // Известия вузов. Строительство и архитектура, 1968. № 11. C. $35-39$.

6. Гузеев Р. Н., Сливкер В. И. Обобщенная задача Тимошенко // Строителъная механика и расчет сооружений, 2009. № 1. С. 12-16.

7. Деревянкин Д. В., Сливкер В. И. О конечноэлементных аппроксимациях в задачах устойчивости стержней Тимошенко // Вестник гражданских инженеров, 2008. № 4(17). C. $17-26$.

8. Perelmuter A. V., Slivker V. I. Handbook of Mechanical Stability in Engineering. vol. 2: Stability of Elastically Deformable Mechanical Systems. Hackensack, NJ: World Scientific, 2013. 1143-1150 pp.. doi : 10.1142/9789814383769_fmatter02.

9. Тацій Р., Ушак Т. Метод дискретизації в задачах про втрату стійкості однопрольотних стрижнів зі змінними параметрами // Фізико-математичне моделювання та інформаційні технологї, 2009. №9. С. 107-117 (на украинском), http://dspace.nbuv.gov.ua/ handle/123456789/22090.

10. Сеницкий Ю. Э., Сумин В. А. Расчёт на общую устойчивость крановых стрел переменного и постоянного сечений // Известия вузов. Строительство и архитектура, 1971. №6. С. $12-17$.

11. Завьялова О. Б., Шеин А. И. Применение условного сдвиго-изгибного стержня при расчете рам на устойчивость // Известия вузов. Строительство, 2010. № 1. С. 99-105. 
12. Сеницкий Ю. Э., Лебедь А. Г. Устойчивость сжатых составных стержней со степенным законом изменения жёсткости // Известия вузов. Строительство и архитектуpa, 1974. № 4. С. 36-41.

13. Сеницкий Ю. Э. Об учёте деформаций сдвига при исследовании устойчивости и колебаний составных стержней // Известия вузов. Строительство и архитектура, 1966. № 6. C. $48-52$.

Поступила в редакцию 17/IX/2014; в окончательном варианте - 01/XII/2014; принята в печать - 08/IV/2015.

Vestn. Samar. Gos. Techn. Un-ta. Ser. Fiz.-mat. nauki

[J. Samara State Tech. Univ., Ser. Phys. \& Math. Sci.], 2015, vol. 19, no. 2, pp. 341-357

ISSN: 2310-7081 (online), 1991-8615 (print)

doi: http://dx.doi.org/10.14498/vsgtu1350

MSC: $74 \mathrm{~K} 10$

\title{
OVERALL STABILITY OF COMPRESSED COMPOUND BARS IN VARIABLE CROSS SECTION
}

\author{
Yu. E. Senitsky, A. S. Ishutin
}

Samara State University of Architecture and Civil Engineering, 194, Molodogvardeyskaya st., Samara, 443001, Russian Federation.

\begin{abstract}
The article provides the solution to the resistance problem of centrally compressed compound bars of variable section with power-law hardening. The assumed model of S. Timoshenko's theory is valid for calculating and analyzing the general resistance of tower-type bar systems (towers, masts, trestle supports) under certain conditions of stiffening behavior. Unlike the traditional way, the plastic design of lateral shear is made on the basis of independent equilibrium equations. The article describes the condition under which the traditional approach accepted in the technical rod theory and based on the inner forces correlation is valid. Boundary value problem is formulated on the basis of the resolving quadratic equation (traditional approach) and the equation of fourth order (more general, suggested in the paper, adequate definition). For this purpose the technique associated with the increase of the resolving equation's degree is used. In the first case it is possible to examine only symmetric forms of resistance loss. In the second case both symmetric and asymmetric forms of resistance loss are possible to be examined. Transcendental equation of resistance for different cases of bar's fixing is obtained. The coefficients of the given length are analyzed depending upon the ways of fixing the end sections. The article points out
\end{abstract}

(C) 2015 Samara State Technical University.

Please cite this article in press as:

Senitsky Yu. E., Ishut in A. S. Overall stability of compressed compound bars in variable cross section, Vestn. Samar. Gos. Tekhn. Univ., Ser. Fiz.-Mat. Nauki [J. Samara State Tech. Univ., Ser. Phys. \& Math. Sci.], 2015, vol. 19, no. 2, pp. 341-357. doi: 10.14498/vsgtu1350. (In Russian)

\section{Authors Details:}

Yuri E. Senitsky (Dr. Techn. Sci.; senitskiy@mail.ru; Corresponding Author), Head of Dept., Dept. of Strength of Materials and Structural Mechanics.

Alexander S. Ishutin, Student, Dept. of Strength of Materials and Structural Mechanics. 
that unlike the situation with the bars of solid cross-section it is necessary to take into account the shear strain of the grid in the compound bars of variable stiffness while examining their general buckling resistance.

Keywords: overall stability, bifurcation, lattice bar, shift deformation, various types of fixation, non-autonomous differential equations, degree increment, accurate solution, effective length factor.

doi: http://dx.doi.org/10.14498/vsgtu1350

\section{ORCID}

Yuri E. Senitsky: http://orcid.org/0000-0002-0663-5563

\section{REFERENCES}

1. Dinnik A. N. Prodol'nyi izgib. Teoriia i prilozheniia [Buckling. Theory and Applications]. Moscow, Leningrad, GONTI, 1939, 238 pp. (In Russian)

2. Timoshenko S. P., Gere J. M. Theory of Elastic Stability. New York, McGraw-Hill, 1961, xvi+541 pp.

3. Vol'mir A. S. Ustoichivost' deformirovannykh sistem [Stability of Deformable Systems]. Moscow, Nauka, 1967, 984 pp. (In Russian)

4. Leites S. D. The stability of compressed rods, hardness of which varies according to a power law, Materialy po metallicheskim konstruktsiiam [Materials of metal structures]. Moscow, Stroiizdat, 1962, pp. 13-74 (In Russian).

5. Senitsky Yu. E. Stability of passing columns with variable section loaded by polar forces, Izvestiia vuzov. Stroitel'stvo i arkhitektura, 1968, no. 11, pp. 35-39 (In Russian).

6. Guzeev R. N., Slivker V. I. Appendix H. Generalized Timoshenko problem, Perelmuter A. V., Slivker V. I. Handbook of Mechanical Stability in Engineering, vol. 2, Stability of Elastically Deformable Mechanical Systems. Hackensack, NJ, World Scientific, 2013, pp. 1143-1150. doi: 10.1142/9789814383769_bmatter02.

7. Derevyankin D. V., Slivker V. I. On finite element approximations in the Timoshenko bar stability problems, Vestnik grazhdanskikh inzhenerov, 2008, no.4(17), pp. 17-26 (In Russian).

8. Perelmuter A. V., Slivker V. I. Handbook of Mechanical Stability in Engineering, vol. 2, Stability of Elastically Deformable Mechanical Systems. Hackensack, NJ, World Scientific, 2013. doi: 10.1142/9789814383769_fmatter02.

9. Tatsii R., Ushak T. Method of digitization in problems of resistance loss of single-span rods with variable parameters, Fiziko-matematichne modeliuvannia ta informatsiini tekhnologii, 2009, no. 9, pp. 107-117 (in Ukrainian), http://dspace.nbuv.gov.ua/handle/123456789/ 22090.

10. Senitsky Yu. E., Sumin V. A. Calculation of the overall stability of crane booms with alternating and direct cross sections, Izvestiia vuzov. Stroitel'stvo i arkhitektura, 1971, no. 6, pp. 12-17 (In Russian).

11. Zav'yalova O. B., Shein A. I. Application of conditional shear-bending beam in the calculation of the frames stability, Izvestiia vuzov. Stroitel'stvo, 2010, no. 1, pp. 99-105 (In Russian).

12. Senitsky Yu. E., Lebed' A. G. Stability of compressed composite rods with a power law changes stiffness, Izvestiia vuzov. Stroitel'stvo i arkhitektura, 1974, no.4, pp. 36-41 (In Russian).

13. Senitsky Yu. E. On the account of the shear strain in the study of stability and vibration of composite rods, Izvestiia vuzov. Stroitel'stvo i arkhitektura, 1966, no.6, pp. 48-52 (In Russian). 